\title{
Isolation of a new antibacterial peptide achromosin from Streptomyces achromogenes subsp. achromogenes based on genome mining
}

\author{
Issara Kaweewan $^{1}$, Mayumi Ohnishi-Kameyama ${ }^{2}$ and Shinya Kodani ${ }^{1,3,4}$ \\ The Journal of Antibiotics (2017) 70, 208-211; doi:10.1038/ja.2016.108; published online 7 September 2016
}

Lasso peptides are a class of ribosomally biosynthesized and posttranslationally modified peptides with a common motif of knot structure in the molecule. ${ }^{1}$ The amino group of the $\mathrm{N}$-terminal amino acid forms a peptide bond with side chain carboxyl group of Asp or Glu in the eighth or the ninth position from the $\mathrm{N}$-terminus, resulting in formation of a macrolactam ring. The macrolactam ring looks like a loop of a 'lasso' with a tail of the C-terminal linear peptide that normally locates through the ring. Regarding lasso peptides, a wide variety of biological activities such as anti-HIV, ${ }^{2}$ antimycobacterial, ${ }^{3}$ endothelin type $\mathrm{B}$ receptor antagonist ${ }^{4}$ and prolyl endopeptidase inhibition $^{5}$ were reported. In addition, lasso peptides normally show a stable property against proteolytic, thermal and chemical degradation, which makes lasso peptides attractive in terms of practical application as pharmaceutical reagents.

Lasso peptides derived from actinobacteria have been classified into three main classes on the basis of their $N$-terminal residues and the number of disulfide bridges. ${ }^{1}$ The class I lasso peptides include siamycins I and II, ${ }^{2}$ aborycin ${ }^{6}$ and sviceucin, ${ }^{7}$ which have an internal peptide linkage between $\beta$-carboxyl group residue of Asp9 (ninth amino acid residue from the $\mathrm{N}$-terminus) and the amino residue of Cys1. These peptides commonly have additional two disulfide bridges between Cys1 and Cys13, and Cys7 and Cys19. The class II lasso peptides include anantin, ${ }^{8}$ lariatins, ${ }^{3}$ propeptin, ${ }^{5}$ RES-701- $1,{ }^{4}$ SRO15-2005 ${ }^{9}$ and sungsanpin. ${ }^{10}$ These peptides have an internal peptide linkage between $\beta$-carboxyl residue of Asp8 or Asp9 and the amino residue of Glyl without any disulfide bonds. The class III lasso peptide includes only one peptide named BI-32169. ${ }^{11}$ The peptide BI-32169 has an internal peptide linkage between $\beta$-carboxyl residue of Asp9 and the amino residue of Glyl with one disulfide bond between Cys6 and Cys19.

The lasso peptide microcin J25 was isolated from Escherichia coli, which is regarded as the archetype of lasso peptides. ${ }^{12}$ Its biosynthetic gene cluster consists of four genes including a precursor peptidecoding gene: gene A ( $m c j A)$, two maturation enzymes including gene
B ( $m c j B$, cleavage of leader peptide) and gene $\mathrm{C}(m c j C$, formation of macrolactam ring) and an ATP-binding cassette transporter-coding gene: gene $\mathrm{D}(m c j D) .{ }^{13}$ The protein $\mathrm{McjC}$ was reported to form the macrolactam ring, and the function of the protein $\mathrm{McjB}$ was assigned to cleave off the leader peptide from the precursor peptide by in vitro experiments. ${ }^{14}$ Normally lasso peptide biosynthetic genes in proteobacteria have a corresponding set of the genes, although the transporter gene is optional. ${ }^{1}$ In actinobacteria, lasso peptide biosynthetic genes consist of a similar gene set, except that a maturation enzyme gene $B$ has split-B genes (gene B1 and gene B2). ${ }^{1,15}$ By genome mining, biosynthetic genes of a lasso peptide sviceucin were found on the genome of Streptomyces sviceus, and the lasso peptide was isolated and structure-determined by heterologous expression. ${ }^{7}$ The lasso peptide SRO15-2005 was identified by matrixassisted laser desorption/ionization-time-of-flight tandem mass spectrometry (MALDI-TOF-MS/MS) from the extract of Streptomyces roseosporus, based on genome sequence data. ${ }^{9}$ On the basis of genome mining, a new lasso peptide chaxapeptin was also isolated as a lung cancer invasion inhibitor from Streptomyces leeuwenhoekii. ${ }^{16}$ These results prompted us to find a new lasso peptide from streptomycetes using genome sequence data. By genome search approach, we found new lasso peptide biosynthetic genes on the genome sequence of Streptomyces achromogenes subsp. achromogenes. ${ }^{17}$ The new antibacterial peptide was isolated by chromatographic separation from the culture of S. achromogenes subsp. achromogenes. Here, we describe isolation and structure determination of a new antibacterial peptide named achromosin.

In the genome sequence of Streptomyces achromogenes subsp. achromogenes, ${ }^{17}$ lasso peptide modification enzyme-coding genes (gene C named acrC: WP_063755122.1, acrB2: WP_037654156.1, acrB1: WP_037654159.1, shown in Figure 1a and Supplementary Table S1) were found by blastp similarity search. As the lasso precursor peptide-coding gene was not annotated, we searched for the lasso precursor peptide-coding gene in the close region to the

${ }^{1}$ Graduate School of Integrated Science and Technology, Shizuoka University, Shizuoka, Japan; ${ }^{2}$ Food Research Institute, National Agriculture and Food Research Organization (NARO), Ibaraki, Japan; ${ }^{3}$ College of Agriculture, Academic Institute, Shizuoka University, Shizuoka, Japan and ${ }^{4}$ Graduate School of Science and Technology, Shizuoka University, Shizuoka, Japan

Correspondence: Dr S Kodani, College of Agriculture, Academic Institute, 836 Ohya, Suruga-ku, Shizuoka $422-8529$ Japan.

E-mail: kodani.shinya@shizuoka.ac.jp

Received 15 May 2016; revised 3 July 2016; accepted 30 July 2016; published online 7 September 2016 
a

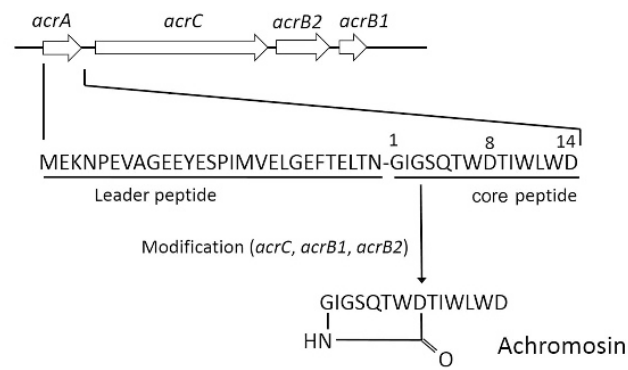

b Achromosin precursor ME-KNPEVAGEEYESPTMVET GEFTELTNGTGS QTWDTIWLWD Chaxapeptin precursor MEPONTELOEEAYEAPSLIEVGEFSEDTLGGSKPLDSELNFE

Figure 1 (a) Gene cluster for biosynthesis of achromosin including four genes (acrA: structural gene, and modification genes: $\operatorname{acrC}$, acrB1 and acrB2), (b) Alignment of amino acid sequences of achromosin and chaxapeptin precursor peptide genes (underlined letters: leader peptide, bold letter: conserved amino acid, arrow: cleavage position).

modification enzyme-coding genes. Upstream of the gene acrC (WP_063755122.1), a new putative precursor peptide-coding gene for new peptide named achromosin (126 base pairs, 42 amino acids, Figure $1 \mathrm{~b}$ ) similar to chaxapeptin ${ }^{16}$ was found from position 72827 to $72952 \mathrm{bp}$ in the genome sequence (GenBank accession number: NZ_JODT01000002.1). On the upstream of 9 residues of the precursor peptide-coding region (72827-72952), Shine-Dalgarno sequence (AGGAGGA) was present. As shown in Figure $1 \mathrm{~b}$, the expected peptide achromosin was deduced to have the amino acid sequence of GIGSQTWDTIWLWD (monoisotopic molecular weight: 1676.7 Da ), after cleaving off the leader peptide at the same position after the conserved motif 'GEFXEXTX' as the biosynthesis of chaxapeptin ${ }^{16}$ (arrow in Figure 1b). The expected monoisotopic molecular weight of achromosin was calculated to be $1658.7 \mathrm{Da}$ considering the loss of $18 \mathrm{Da}$, resulting in macrolactam formation of lasso peptide biosynthesis. The preliminary chemical investigation of S. achromogenes subsp. achromogenes $\mathrm{NBRC} 12735^{\mathrm{T}}$ indicated that the expected peptide was present in the methanol extract of aerial hyphae and spore cells by high-performance liquid chromatography (HPLC) and electrospray ionization mass spectrometry (ESI-MS). Thus, cultivation of $S$. achromogenes subsp. achromogenes was performed in a large scale to obtain enough amount of the peptide for structure determination. After 7 days of cultivation, cells of spore and aerial hyphae were harvested by a steel spatula. The cells were extracted with double volume of methanol $(\mathrm{MeOH})$, followed by centrifugation. After condensation using rotary evaporation, the extract was subjected to open-column chromatography using hydrophobic resin (CHP$-20 \mathrm{P}$ ), eluted with $10 \%, 60 \%$ and $100 \% \mathrm{MeOH}$. The expected peptide achromosin was detected in $100 \% \mathrm{MeOH}$ fraction by HPLC (Supplementary Figure S1) and ESI-MS analysis (Supplementary Figure S2). The ESI-MS analysis of the peptide gave an ion peak at $\mathrm{m} / z 1659.7$ for $[\mathrm{M}+\mathrm{H}]^{+}$. The $100 \% \mathrm{MeOH}$ fraction was repeatedly subjected to HPLC purification to give pure achromosin.

The molecular formula of achromosin was established to be $\mathrm{C}_{79} \mathrm{H}_{106} \mathrm{~N}_{18} \mathrm{O}_{22}$ by accurate mass analysis using the ESI Fouriertransform ion cyclotron resonance mass spectrometry, as $[\mathrm{M}+2 \mathrm{H}]^{2+}$ was observed at $m / z 830.3941$ corresponding to $\mathrm{C}_{79} \mathrm{H}_{108} \mathrm{~N}_{18} \mathrm{O}_{22}$ whose calculated value was 830.3937 . The amino acid composition analysis was performed on achromosin following the reported method. ${ }^{18}$ The amino acid content analysis on achromosin afforded the relative molar ratios of the constituent amino acids (2 moles each of Asp/Asn, Gly, Ile and Thr, and 1 mole each of Glu/Gln, Leu and Ser), as shown in
Supplementary Figure S3. Nuclear magnetic resonance analysis using dimethyl sulfoxide- $d_{6}$ as a solvent was not possible due to ambiguous broad peaks in the nuclear magnetic resonance spectrum. To obtain peptide sequence, MALDI-TOF-MS/MS analysis on achromosin was accomplished. As a result, the product ions from achromosin at $\mathrm{m} / \mathrm{z}$ 1659 were of $b$-series peptides, $b 8$ - $b 13$ (Figure $2 \mathrm{a}$ and Supplementary Table S2), which indicated that the sequence of TIWLWD was the $\mathrm{C}$-terminus tail sequence. Macrolactam ring structure was reported not to give fragment ions, ${ }^{9}$ thus we proposed the structure of achromosin to be shown in Figure 2a, based on the amino acid sequence of precursor peptide gene. To confirm the amino acid sequence in the macrolactam ring, C-terminal peptide bonds of tryptophans were cleaved by BNPS-skatole. After BNPS-skatole reaction, the cleaved achromosin (BNPS-achromosin) was purified by HPLC separation. ESI-TOF-MS analysis on BNPS-achromosin gave an ion peak at $m / z 1291.5$ for $[\mathrm{M}+\mathrm{H}]^{+}$(Supplementary Figure S4). The molecular formula of BNPS-achromosin was clarified to be $\mathrm{C}_{58} \mathrm{H}_{78} \mathrm{~N}_{14} \mathrm{O}_{20}$ by the accurate mass analysis. That is, $[\mathrm{M}+2 \mathrm{H}]^{2+}$ was observed at $m / z 646.2832$ corresponding to $\mathrm{C}_{58} \mathrm{H}_{80} \mathrm{~N}_{14} \mathrm{O}_{20}$ whose calculated value was 646.2831 . By the reaction of BNPS-skatole the Trp residue in a peptide is oxidized and transformed to 3-oxindole with a spirolactone, which increases the molecular weight due to the addition of two oxygens by $32 \mathrm{Da}$. As shown in Figure 2b, the MALDITOF-MS/MS of the cleaved achromosin gave the sequence of the peptide with one $\mathrm{N}$-terminus and two C-terminal ends (Supplementary Table S3). The product ions of $b 1, b 2$ and $b 3$ supported the sequence of $\mathrm{DTIW}^{\star}$ and $b 4$ ion especially indicated that $\operatorname{Trp}$ at C-terminus was oxidized (indicated with asterisk, Figure $2 \mathrm{~b}$ ). The product ions of $y 2$ to $y 7$ supported the sequence of GIGSQTW $^{\star}$ (Figure 2b). Above all, the structure of achromosin was proposed to be a peptide with the sequence of GIGSQTWDTIWLWD having one macrolactam ring which was formed by peptide bond between amino residue of Gly1 and $\beta$-carboxyl residue of Asp8 (Figure 2a). The structure of achromosin did not include any disulfide bridge in the molecule, which classified achromosin into class II lasso peptide.

The antimicrobial activity of achromosin was tested using a paper disk agar-diffusion assay against microorganisms (bacterial strains including E. coli, Pseudomonas aeruginosa, Serratia marcescens, Bacillus subtilis, Staphylococcus aureus, Micrococcus luteus and Streptomyces antibioticus; Yeast strains including Saccharomyces cerevisiae, Schizosaccharomyces pombe, Kloeckera apiculata; fungi strains including Aspergillus niger, Aspergillus oryzae and Mucor hiemalis). At the dosage of $10 \mu \mathrm{g}$ per disk, achromosin showed an inhibitory zone of $11 \mathrm{~mm}$ diameter against $M$. luteus (Supplementary Figure S5). On the other hand, achromosin did not show any inhibitory activity against the other testing microorganisms at the same dosage.

Biosynthetic gene clusters of lasso peptides of actinobacteria have been identified for lasso peptides including lariatin, ${ }^{19}$ SRO15-2005, ${ }^{9}$ lassomycin, ${ }^{20}$ sviceucin, ${ }^{7}$ chaxapeptin ${ }^{16}$ and streptomonomicin. ${ }^{21}$ The biosynthetic gene cluster of chaxapeptin consisted of four genes including $c p t A, c p t C, c p t B 1$ and $c p t B 2 .{ }^{16}$ Interestingly, the gene cluster of chaxapeptin lacked of transporter gene that often exists in the lasso peptide biosynthetic gene cluster. The gene $c p t A$ encoded chaxapeptin precursor peptide, and the three genes including $c p t C, c p t B 1$ and $c p t B 2$ were proposed to be involved in macrolactam formation and leader peptide cleavage. The amino acid sequence of precursor peptide gene acrA which was found on the genome of $S$. achromogenes subsp. achromogenes ${ }^{17}$ showed high similarity with that of $c p t A$ ( $46 \%$ identity, $68 \%$ positive matches). By reference to chaxapeptin biosynthetic genes, we assigned the biosynthetic gene cluster for achromosin, 
a
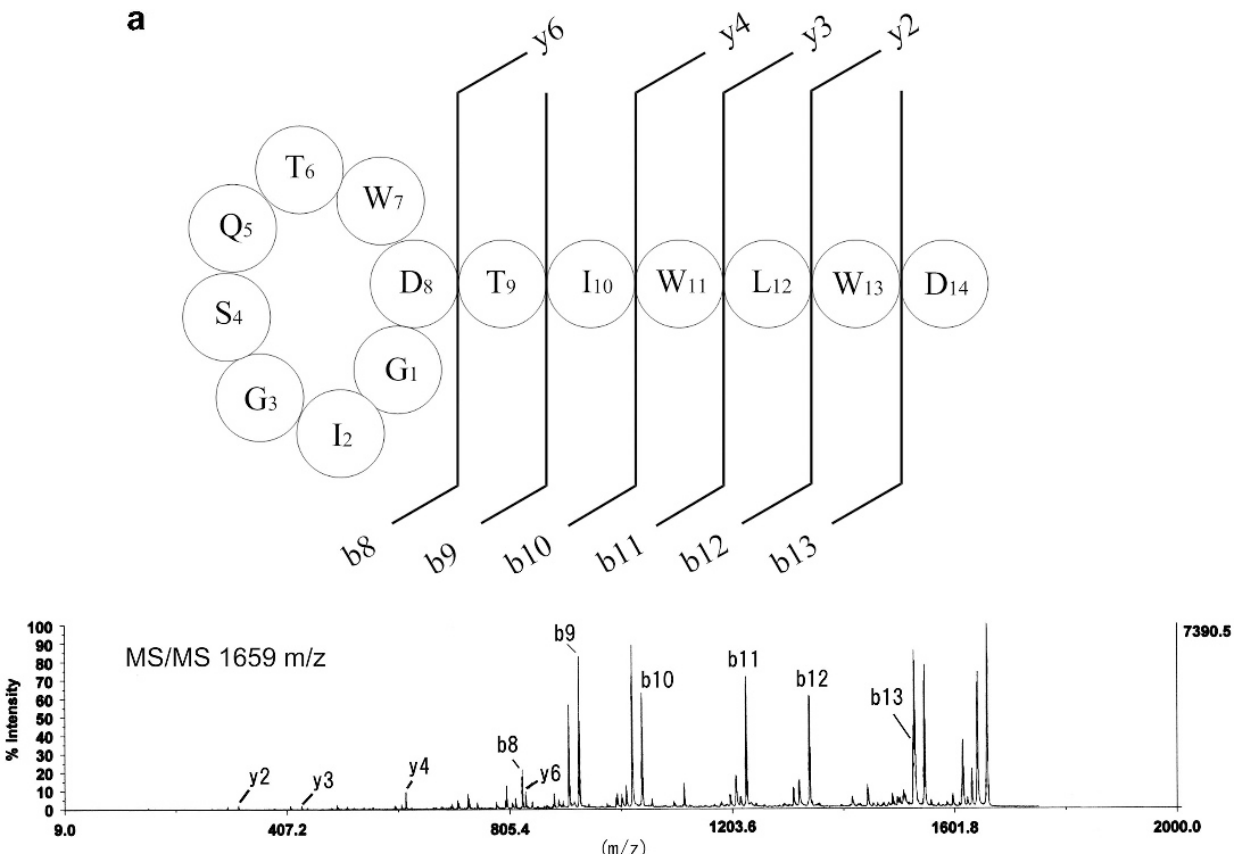

b
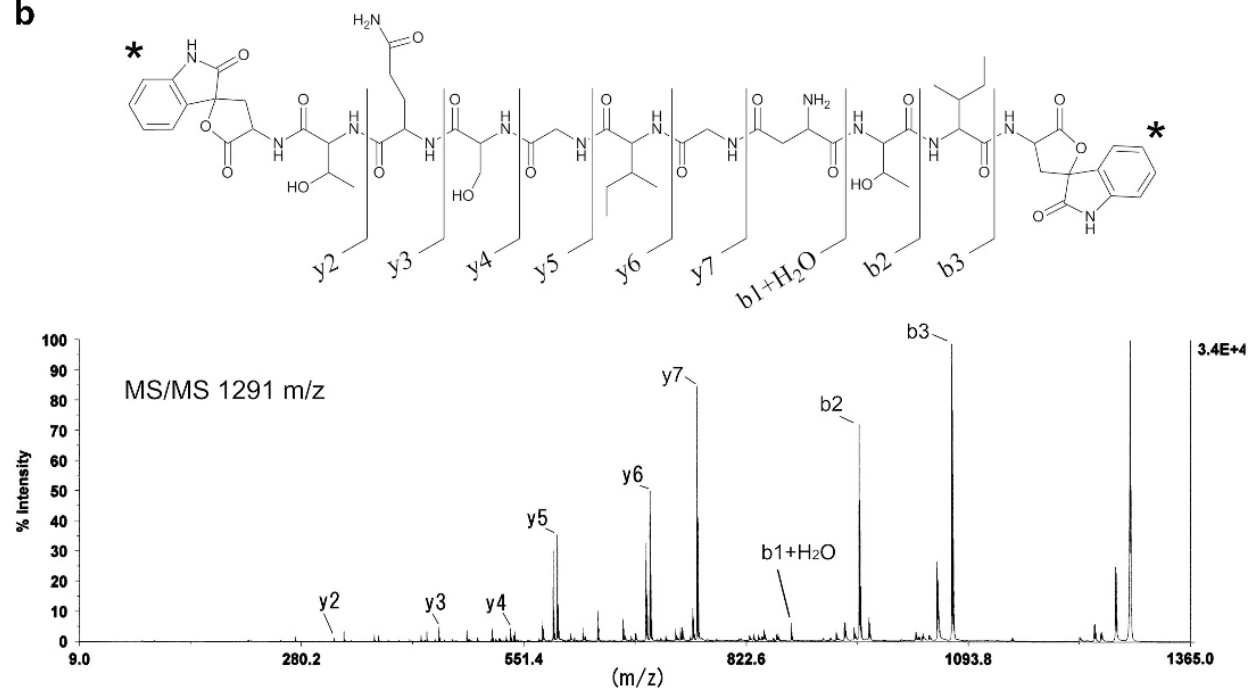

Figure 2 (a) MALDI-TOF-MS/MS analysis of achromosin, (b) MALDI-TOF-MS/MS analysis of BNPS-skatole cleaved achromosin (the oxidized Trp residue is marked by an asterisk).

which have four genes, acrA (annoted in this study, 42 aa), acr $C$ (WP_063755122.1, 616 aa), acrB2 (WP_037654156.1, 150 aa) and acrB1 (WP_037654159.1, 95 aa) in this order with all the same direction (Figure 1a). Interestingly, there was no transport proteincoding genes near the gene cluster. The lack of transport gene was also reported in the chaxapeptin gene cluster. ${ }^{16}$ On the basis of the similarity of each gene, we proposed the functions of the genes as shown in Figure 1a. The gene acrA encoded the precursor of achromosin and the genes including $\operatorname{acr} C, \operatorname{acrB1}$ and $a c r B 2$ were proposed to be modification enzymes to give the mature lasso peptide. The gene acrC encoded putative asparagine synthase possibly responsible for formation of the Gly1-Glu8 amide bond, which showed high similarity to $c p t C$ by using a BLAST homology search (37\% identity, $51 \%$ positive matches). The amino acid sequence of acrB2 showed high similarity to that of $c p t B 2$ by using a BLAST homology search (55\% identity, $69 \%$ positive matches) and the amino acid sequence of acrB1 showed high similarity to that of $c p t B 1$ by using a BLAST homology search (40\% identity, $54 \%$ positive matches). Above all, the biosynthetic genes of achromosin showed the similarity to those of chaxapeptin.

So far, no similar peptide has been found by the blastp search, which indicates the novelty of achromosin. As shown in Figure 1b, the amino acid sequence of core peptide is different even from that of chaxapeptin, the closest lasso peptide. The lasso peptide in class II were reported to have a wide variety of biological activities such as antimycobacterial, ${ }^{3}$ endothelin type B receptor antagonist ${ }^{4}$ and prolyl endopeptidase inhibition ${ }^{5}$. In this paper, the antimicrobial activity of achromosin was tested. Further bioactivity tests may lead to the discovery of additional activities of achromosin. In addition, the biosynthetic genes of achromosin were identified from the genome of S. achromogenes subsp. achromogenes, which will lead to genetic engineering using the gene cluster to create mutated lasso peptide 
based on achromosin by heterologous expression. The modified peptides with more potent antibacterial activity may be produced by altering the amino acid sequence of achromosin by further genetic engineering experiments.

\section{CONFLICT OF INTEREST}

The authors declare no conflict of interest.

\section{ACKNOWLEDGEMENTS}

This study was supported by the Japan Society for the Promotion of Science by Grants-in-aids (grant number 25350964). We thank Ms Tomoko Satoh (Bruker Daltonics) for her technical assistance in the MS analysis.

1 Li, Y., Zirah, S. \& Rebuffat, S. Lasso Peptides: Bacterial Strategies to Make and Maintain Bioactive Entangled Scaffolds (Springer, 2015).

2 Detlefsen, D. J. et al. Siamycins I and II, new anti-HIV-1 peptides: II. Sequence analysis and structure determination of siamycin I. J. Antibiot. 48, 1515-1517 (1995).

3 Iwatsuki, M. et al. Lariatins, antimycobacterial peptides produced by Rhodococcus sp. K01-B0171, have a lasso structure. J. Am. Chem. Soc. 128, 7486-7491 (2006).

4 Morishita, Y. et al. RES-701-1, a novel and selective endothelin type B receptor antagonist produced by Streptomyces sp. RE-701. I. Characterization of producing strain, fermentation, isolation, physico-chemical and biological properties. J. Antibiot. 47, 269-275 (1994).

5 Kimura, K. et al. Propeptin, a new inhibitor of prolyl endopeptidase produced by Microbispora. I. Fermentation, isolation and biological properties. J. Antibiot. 50, 373-378 (1997).

6 Potterat, 0. et al. Tricyclic 21-peptide antibiotic isolated from Streptomyces griseoflavus. Liebigs. Ann. 1994, 741-743 (1994).
$7 \mathrm{Li}, \mathrm{Y}$. et al. Characterization of sviceucin from Streptomyces provides insight into enzyme exchangeability and disulfide bond formation in lasso peptides. ACS. Chem. Biol. 10, 2641-2649 (2015).

8 Weber, W. et al. Anantin-a peptide antagonist of the atrial natriuretic factor (ANF). I. Producing organism, fermentation, isolation and biological activity. J. Antibiot. 44, 164-171 (1991).

9 Kersten, R. D. et al. A mass spectrometry-guided genome mining approach for natural product peptidogenomics. Nat. Chem. Biol. 7, 794-802 (2011).

$10 \mathrm{Um}, \mathrm{S}$. et al. Sungsanpin, a lasso peptide from a deep-sea streptomycete. J. Nat. Prod. 76, 873-879 (2013).

11 Potterat, 0. et al. BI-32169, a bicyclic 19-peptide with strong glucagon receptor antagonist activity from Streptomyces sp. J. Nat. Prod. 67, 1528-1531 (2004).

12 Salomon, R. A. \& Farias, R. N. Microcin 25, a novel antimicrobial peptide produced by Escherichia coli. J. Bacteriol. 174, 7428-7435 (1992).

13 Solbiati, J. O. et al. Genetic analysis of plasmid determinants for microcin J25 production and immunity. J. Bacteriol. 178, 3661-3663 (1996).

14 Yan, K. P. et al. Dissecting the maturation steps of the lasso peptide microcin J25 in vitro. Chembiochem. 13, 1046-1052 (2012).

15 Burkhart, B. J. et al. A prevalent peptide-binding domain guides ribosomal natural product biosynthesis. Nat. Chem. Biol. 11, 564-570 (2015).

16 Elsayed, S. S. et al. Chaxapeptin, a lasso peptide from extremotolerant Streptomyces leeuwenhoekii strain C58 from the hyperarid atacama desert. J. Org. Chem. 80, 10252-10260 (2015).

$17 \mathrm{Ju}, \mathrm{K}$. S. et al. Discovery of phosphonic acid natural products by mining the genomes of 10,000 actinomycetes. Proc. Natl Acad. Sci. USA 112, 12175-12180 (2015).

18 Heinrikson, R. L. \& Meredith, S. C. Amino acid analysis by reverse-phase high-performance liquid chromatography: precolumn derivatization with phenylisothiocyanate. Anal. Biochem. 136, 65-74 (1984).

19 Inokoshi, J. et al. Molecular cloning of the gene cluster for lariatin biosynthesis of Rhodococcus jostii K01-B0171. Appl. Microbiol. Biotechnol. 95, 451-460 (2012).

20 Gavrish, E. et al. Lassomycin, a ribosomally synthesized cyclic peptide, kills mycobacterium tuberculosis by targeting the ATP-dependent protease CIpC1P1P2. Chem. Biol. 21, 509-518 (2014).

21 Metelev, M. et al. Structure, bioactivity, and resistance mechanism of streptomonomicin, an unusual lasso peptide from an understudied halophilic actinomycete. Chem. Biol. 22, 241-250 (2015).

Supplementary Information accompanies the paper on The Journal of Antibiotics website (http://www.nature.com/ja) 